\title{
hCINAP serves a critical role in hypoxia-induced cardiomyocyte apoptosis via modulating lactate production and mitochondrial-mediated apoptosis signaling
}

\author{
HEBING XIE ${ }^{1,2}$, GANG XU ${ }^{1,2}$, YUQI GAO ${ }^{1,2}$ and ZHIBIN YUAN ${ }^{1,2}$ \\ ${ }^{1}$ Institute of Medicine and Hygienic Equipment for High Altitude Region, \\ ${ }^{2}$ Key Laboratory of Extreme Environmental Medicine of Ministry of Education, College of High Altitude Military Medicine, \\ Army Medical University (Third Military Medical University), Chongqing 400037, P.R. China
}

Received April 19, 2020; Accepted October 30, 2020

DOI: $10.3892 / \mathrm{mmr} .2020 .11748$

\begin{abstract}
Acute myocardial infarction (AMI) is a major cause of heart failure and is associated with insufficient myocardial oxygen supply. However, the molecular mechanisms underlying hypoxia-induced cardiomyocyte apoptosis are not completely understood. In the present study, the role of human coilin interacting nuclear ATPase protein (hCINAP) in cardiomyocytes was investigated. AC16 cells were divided into the following four groups: i) Small interfering (si) RNA-control (Ctrl); (ii) siRNA-hCINAP; (iii) empty vector; and (iv) hCINAP-Flag. Protein expression was assessed using western blotting. MTT and apoptosis assays were conducted to detect cell viability and apoptosis, respectively. CCK8 assays and apoptosis assays were used to detect cell viability and apoptosis, respectively. hCINAP promoter activity was examined by luciferase reporter assay. hCINAP expression was induced in a hypoxia-inducible factor- $1 \alpha$-dependent manner under hypoxic conditions. Compared with the siRNA-Ctrl group, hCINAP knockdown inhibited apoptosis, whereas compared with the vector group, hCINAP overexpression increased apoptosis under hypoxic conditions. Mechanistically, compared with the siRNA-Ctrl group, hCINAP knockdown decreased hypoxia-induced lactate accumulation via regulating lactate dehydrogenase A activity. Moreover, the results indicated that hCINAP was associated with mitochondrial-mediated apoptosis via Caspase signaling. Collectively, the present study suggested that hCINAP was an important regulator
\end{abstract}

Correspondence to: Professor Zhibin Yuan or Professor Yuqi Gao, Institute of Medicine and Hygienic Equipment for High Altitude Region, College of High Altitude Military Medicine, Army Medical University (Third Military Medical University), 30 Gaotanyan Main Road, Shapingba, Chongqing 400037, P.R. China

E-mail: zhibinyuan@sina.com

E-mail: gaoy66@yahoo.com

Key words: human coilin interacting nuclear ATPase protein, lactate, hypoxia, lactate dehydrogenase A, apoptosis in hypoxia-induced apoptosis and may serve as a promising therapeutic target for AMI.

\section{Introduction}

Heart failure caused by acute myocardial infarction (AMI) is the primary pathological cause of death and disability worldwide $(1,2)$. Coronary occlusion deprives cardiomyocytes of oxygen, leading to cardiac dysfunction during AMI (3). Cardiomyocytes are terminally differentiated cells, thus preserving cardiomyocytes from hypoxia-induced apoptosis has been proposed as a therapeutic strategy for AMI (4). Previous studies reported that hypoxia induces dissipation of mitochondrial membrane potential and initiates mitochondrial-mediated apoptosis, thus leading to an increase in the number of dysfunctional mitochondria $(5,6)$. Bcl-2 family proteins are involved in the regulation of mitochondrial apoptosis via regulating cytochrome $\mathrm{C}$ release from mitochondria (7). Additionally, hypoxia-induced reactive oxygen species (ROS) generation promotes the release of cytochrome $\mathrm{C}$ into the cytoplasm, resulting in DNA damage, oxidative stress, apoptosis and mitochondrial damage $(8,9)$. Hypoxia and an altered redox balance contribute to the mechanisms regulating the response to tissue damage (10). For example, excessive production of ROS initiates the intrinsic apoptosis signaling pathway in testicular tissue cells, leading to testicular tissue damage in individuals with varicocele $(11,12)$. However, the regulatory mechanisms underlying hypoxia-induced mitochondrial-mediated apoptosis in cardiomyocytes are complex and are not completely understood.

Activation of hypoxia-inducible factor-1 (HIF-1) transcription factor serves a critical role in the adaptive responses to hypoxia (13). HIF-1 is a heterodimeric transcription factor that contains two subunits, including HIF-1 $\alpha$ and HIF-1 $\beta$ /aryl hydrocarbon receptor nuclear translocator (14). HIF-1 $\alpha$ is hydroxylated by prolyl hydroxylases domain (PHD) enzymes on two conserved proline residues under normoxic conditions, which is recognized by von Hippel-Lindau, an E3 ubiquitin ligase that ubiquitinates HIF-1 $\alpha$. Under hypoxic conditions, inhibition of PHD enzymes leads to HIF- $1 \alpha$ accumulation and nuclear translocation (15). Upregulation of HIF-1 target genes 
is involved in oxygen transport, glycolytic metabolism, lactate production and secretion, cell death and other processes that can affect cell survival in hypoxia $(16,17)$.

During hypoxia, increased glycolysis causes a surge in lactate production (18). Increasing evidence indicates that elevation of lactate production is associated with myocardial infarction and volume overload $(18,19)$. Lactate dehydrogenase (LDH) serves a crucial role in glucose metabolism and the Warburg effect (20). Lactate dehydrogenase A (LDHA) and lactate dehydrogenase $\mathrm{B}$ (LDHB) have been reported to catalyze the same reaction, the conversion of pyruvate to lactate at the end of glycolysis (21). Under anaerobic conditions, LDHA has a higher affinity for pyruvate, preferentially converting pyruvate to lactate and NADH to NAD ${ }^{+}$. When oxygen supplies are sufficient, LDHB has a higher affinity for lactate, preferentially converting lactate to pyruvate and $\mathrm{NAD}^{+}$to NADH (22). Moreover, hypoxia-induced elevation of LDHA expression can promote tumor cell proliferation (23)

Human coilin interacting nuclear ATPase protein (hCINAP) is highly conserved and ubiquitously expressed in different eukaryotes (24). Nucleoside-triphosphatase, a yeast homolog of hCINAP, is required for yeast growth and 18S rRNA maturation $(21,25)$. hCINAP serves as a critical regulator of the $40 \mathrm{~S}$ ribosomal protein S14 (RPS14)/human double minute 2 (HDM2)/p53 signaling pathway by regulating the interaction between RPS14 and HDM2 in 293T cells (26). hCINAP knockdown can cause defects in the formation of Cajal bodies, histone transcription and cell viability (27). In acute myeloid leukemia model mice, hCINAP knockdown results in increased cell death (28). Furthermore, hCINAP-depleted cells display increased Caspase- 3 activities, indicating that apoptosis is one of the reasons for decreased cell viability (28). hCINAP knockdown inhibits colorectal cancer stem cell invasion and self-renewal via modulating the activation of LDHA (29). Collectively, the aforementioned studies indicate that hCINAP serves a critical role in biological processes; however, the role of hCINAP in hypoxia-induced apoptosis of cardiomyocytes is not completely understood.

The aim of the present study was to investigated the role of hCINAP in hypoxia-induced apoptosis and it demonstrated that hCINAP expression was induced under hypoxic conditions in cardiomyocytes and that hCINAP may serve as a regulator for oxygen-dependent and lactate regulation of hypoxia responses.

\section{Materials and methods}

Cell culture. AC16 cells (a human cardiomyocyte cell line; ATCC) were cultured in DMEM (Gibco; Thermo Fisher Scientific, Inc.) supplemented with $10 \%$ FBS (Gibco; Thermo Fisher Scientific, Inc.) and $1 \%$ penicillin-streptomycin at $37^{\circ} \mathrm{C}$ with $5 \% \mathrm{CO}_{2}$. For hypoxia treatment, cells were cultured with $1 \%$ oxygen, $5 \% \mathrm{CO}_{2}$ and $94 \% \mathrm{~N}_{2}$ for the indicated time points in a hypoxia chamber (Billups-Rothenberg, Inc.).

PCR protocol. The synthesized cDNA was diluted to $15 \mathrm{ng} / \mu 1$ as cDNA template. hCINAP cDNA was amplified by PCR conditions of initial denaturation at $95^{\circ} \mathrm{C}$ for $3 \mathrm{~min}$ followed by 30 cycles of $95^{\circ} \mathrm{C}$ for $30 \mathrm{sec}, 60^{\circ} \mathrm{C}$ for $20 \mathrm{sec}, 72^{\circ} \mathrm{C}$ for $1 \mathrm{~min}$, and final elongation at $72^{\circ} \mathrm{C}$ for 5 min with Platinum II
Hot-Start Green PCR Master Mix (cat. no. 14001012; Thermo Fisher Scientific, Inc.). To construct the hCINAP promoter constructs, genomic DNA was extracted using PureLink Genomic DNA kit (cat. no. K182002; Invitrogen; Thermo Fisher Scientific, Inc.). Briefly, AC16 cells $\left(4 \times 10^{6}\right)$ were lysed with $200 \mu \mathrm{l}$ genomic lysis buffer (cat. no. K182302; Invitrogen; Thermo Fisher Scientific, Inc.) supplied with $20 \mu$ l Proteinase K (cat. no. 25530049; Invitrogen; Thermo Fisher Scientific, Inc.) and $20 \mu 1$ RNase A (cat. no. 12091021; Invitrogen; Thermo Fisher Scientific, Inc.) at $55^{\circ} \mathrm{C}$ for 10 min followed by the addition of $200 \mu 195 \%$ ethanol to the lysate. The lysate was added to a PureLink Spin Column (cat. no. K210012; Invitrogen, Thermo Fisher Scientific, Inc.) and spun for $1 \mathrm{~min}$ at $10,000 \mathrm{x} \mathrm{g}$ at room temperature. DNA fluid was obtained by centrifugation $(10,000 \mathrm{x} \mathrm{g}$ for $2 \mathrm{~min}$ at room temperature) using $500 \mu \mathrm{l}$ wash buffer and $100 \mu \mathrm{l}$ elution buffer. hCINAP promoter ( $-2350 \mathrm{bp}$ to $+50 \mathrm{bp})$ was amplified from genomic DNA by PCR conditions of initial denaturation at $95^{\circ} \mathrm{C}$ for $3 \mathrm{~min}$ followed by 30 cycles of $95^{\circ} \mathrm{C}$ for $30 \mathrm{sec}, 62^{\circ} \mathrm{C}$ for $20 \mathrm{sec}, 72^{\circ} \mathrm{C}$ for $30 \mathrm{~s}$, and final elongation at $72^{\circ} \mathrm{C}$ for 5 min with Platinum II Hot-Start Green PCR Master Mix (cat. no. 14001012; Thermo Fisher Scientific, Inc.). The PCR amplification conditions for truncated hCINAP promoter constructs were the same as those employed in amplification of full-length hCINAP promoter. A $1 \%$ agarose gel and ethidium bromide (cat. no. 15585011; Thermo Fisher Scientific, Inc.) were used for agarose gel electrophoresis. The primer list for PCR is presented in Table I.

Plasmids and reagents. The cDNA of human hCINAP was amplified by PCR and cloned into the pB513B-Flag empty vector (cat. no. 5619; Biovector NTCC, Inc.) to generate the pB513B-hCINAP-Flag vector. The coding sequence of HIF-1 $\alpha$ was cloned in-frame with an ATG start codon into the pcDNA 6.0 expression vector (cat. no. 3791; Biovector NTCC, Inc.) to generate the pcDNA-HIF-1 $\alpha$ vector. For LDHA inhibition, cells were treated with $10 \mu \mathrm{M}$ LDHA inhibitor FX11 (MedChemExpress) and incubated at $37^{\circ} \mathrm{C}$ for $24 \mathrm{~h}$. For lactate treatment, cells were treated with $20 \mathrm{mM}$ lactate (Sigma-Aldrich; Merck KGaA) and incubated at $37^{\circ} \mathrm{C}$ for $24 \mathrm{~h}$. Cytoplasmic and mitochondrial protein fractions were prepared using the Cytoplasmic and Mitochondrial Protein Extraction kit (cat. no. G007-1-1; Nanjing Jiancheng Bioengineering Institute). Caspase-9 and Caspase-3 activities were measured using Caspase-9 Activity Assay kit (cat. no. C1157; Beyotime Institute of Biotechnology) and Caspase-3 Activity Assay kit (cat. no. C1115; Beyotime Institute of Biotechnology) according to the manufacturer's protocol. Lactate production was measured using a Lactate Assay kit (cat. no. BC2230; Beijing Solarbio Science \& Technology Co., Ltd.), according to the manufacturer's protocol. LDHA enzyme activity was measured using a LDHA Assay kit (cat. no. BC0680; Beijing Solarbio Science \& Technology Co., Ltd.), according to the manufacturer's protocol.

Chromatin-immunoprecipitation (ChIP). ChIP assays were performed using a Simple ChIP PlusEnzymatic Chromatin IP kit (Agarose Beads; cat. no. 9004; Cell Signaling Technology, Inc.) according to the manufacturer's protocol. Briefly, AC16 cells $\left(4 \times 10^{6}\right)$ were seeded into $10-\mathrm{cm}$ dishes and allowed to 
Table I. Sequences of primers used in the present study.

\begin{tabular}{|c|c|c|}
\hline Primer & Sequence $\left(5^{\prime} \rightarrow 3\right)$ & Primer purpose \\
\hline GAPDH & $\begin{array}{l}\text { F: TCCTGGTATGACAACGAAT } \\
\text { R: GGTCTCTCTCTTCCTCTTG }\end{array}$ & RT-qPCR \\
\hline hCINAP & $\begin{array}{l}\text { F: GTTGGTTCAGTTACTAGCAGACC } \\
\text { R: CCTTTGACCTGTGAGGGACATG }\end{array}$ & RT-qPCR \\
\hline hCI-pm & $\begin{array}{l}\text { F: CTCGAGAATCTTGGCCCCTTTCCTCT } \\
\text { R: GGTACCGCCCTTCGCTTGCGCC }\end{array}$ & Luciferase reporter assay \\
\hline hCI-pm1 & $\begin{array}{l}\text { F: CTCGAGAATCTTGGCCCCTTTCCTCTATA } \\
\text { R: GGTACCGGATTTGCGAGCTCAACCC }\end{array}$ & Luciferase reporter assay \\
\hline hCI-pm2 & $\begin{array}{l}\text { F: CTCGAGCGTTTCAAAAGGTATACAGGTGG } \\
\text { R: GGTACCGCCCTTCGCTTGCGCCGA }\end{array}$ & Luciferase reporter assay \\
\hline hCI-pm3 & $\begin{array}{l}\text { F: CTCGAGCGTTTCAAAAGGTATACAGGTGG } \\
\text { R: GGTACCGGATTTGCGAGCTCAACCC }\end{array}$ & Luciferase reporter assay \\
\hline hCI-pm-4 & $\begin{array}{l}\text { F: CTCGAGCGTTTCAAAAGGTATACAGGTGG } \\
\text { R: GGTACCTTGTTTTGAGACGGAGTCTCAC }\end{array}$ & Luciferase reporter assay \\
\hline hCI-pm-5 & $\begin{array}{l}\text { F: CTCGAGGGTGAAACCCCGTCTCTATTAA } \\
\text { R: GGTACCGGATTTGCGAGCTCAACCC }\end{array}$ & Luciferase reporter assay \\
\hline hCINAP & $\begin{array}{l}\text { F: CGTTTCAAAAGGTATACAGGTGG } \\
\text { R: TTAATAGAGACGGGGTTTCACC }\end{array}$ & ChIP-qPCR \\
\hline
\end{tabular}

F, forward; R, reverse; hCINAP, human coilin interacting nuclear ATPase protein; RT-qPCR, reverse transcription-quantitative PCR; ChIP, chromatin-immunoprecipitation; hCI-pm, hCINAP-promoter.

adhere overnight. Following treatment, cells were fixed with $1 \%$ formaldehyde at room temperature for $10 \mathrm{~min}$, followed by incubating with ChIP lysis buffer plus protease inhibitors (PIC) on ice for $10 \mathrm{~min}$. Next the lysates were incubated at $37^{\circ} \mathrm{C}$ for $20 \mathrm{~min}$ with micrococcal nuclease (cat. no. 10011; Cell Signaling Technology, Inc.) to digest the chromatin DNA. Immunoprecipitation was performed using an $\operatorname{IgG}(1 \mu \mathrm{g}$; cat. no. 2729; Cell Signaling Technology, Inc.) or HIF-1 $\alpha$ antibody ( $1 \mu \mathrm{g}$; cat. no. NB100-105; Novus Biologicals, LLC) and ChIP-Grade Protein G Agarose Beads (cat. no. 9007; Cell Signaling Technology, Inc.) incubated at $4^{\circ} \mathrm{C}$ for $2 \mathrm{~h}$. Subsequently, chromatin complexes were eluted from the beads using ChIP elution buffer as described in manufacturer's instructions. ChIP DNA was purified with a DNA purification columns (cat. no. 10010; Cell Signaling Technology, Inc.). Immunoprecipitation was used as templates for Chip-qPCR using appropriate primers. Primer sequences for ChIP-qPCR are listed in Table I. ChIP-qPCR were performed as previously described (30).

Western blotting. Total protein was extracted from cells using RIPA buffer (Beijing Solarbio Science \& Technology Co., Ltd.) with protease inhibitor cocktail and protein phosphatase inhibitor (Beijing Solarbio Science \& Technology Co., Ltd.). Protein concentration was determined by Pierce BCA Protein Assay kit (cat. no. 23227; Thermo Fisher Scientific, Inc.). Total Proteins (30 $\mu \mathrm{g})$ were separated via SDS-PAGE $(8 \%)$ and transferred onto PVDF membranes. After blocking with 5\% BSA (cat. no. A8010; Beijing Solarbio Science \& Technology Co., Ltd.) at room temperature for $1 \mathrm{~h}$ and the membranes were incubated at $4^{\circ} \mathrm{C}$ overnight with the following primary antibodies in 5\% BSA: HIF-1 $\alpha$ (1:2,000; cat. no. NB100-105; Novus Biologicals, LLC), hCINAP (1:1,000; cat. no. ab192653; Abcam), $\beta$-actin (1:2,000; cat. no. 3700S; Cell Signaling Technology, Inc.), $\beta$-tubulin (1:3,000; cat. no. $2146 \mathrm{~S}$; Cell Signaling Technology, Inc.), phosphorylated LDHA (1:500; cat. no. 8176S; Cell Signaling Technology, Inc.), Flag-tag (1:3,000; cat. no. 14793S; Cell Signaling Technology, Inc.), LDHA (1:3,000; cat. no. 2012S; Cell Signaling Technology, Inc.), cytochrome C (1:2,000; cat. no. 4280S; Cell Signaling Technology, Inc.), hsp60 (1:1,000; cat. no. 611563; BD Pharmingen; BD Biosciences), monocarboxylate transporter 1 (MCT1; 1:2,000; cat. no. 20139-1-AP; ProteinTech Group, Inc.). Subsequently, the membranes were incubated with HRP-linked anti-mouse IgG (1:8,000; cat. no. 7076P2; Cell Signaling Technology, Inc.) and HRP-linked anti-rabbit IgG (1:5,000; cat. no. 7074P2; Cell Signaling Technology, Inc.) secondary antibodies at room temperature for $1 \mathrm{~h}$. To visualize the images, ECL reagent (cat. no. WBKLS0050; EMD Millipore) was applied and the images were captured by CCD camera (LAS-4000; Fujifilm Life Science). Protein expression was semi-quantified using ImageLab software version 5.2.1. (Bio-Rad Laboratories, Inc.) with $\beta$-actin or $\beta$-tubulin as the loading control.

$R T-q P C R$. Total RNA was extracted and purified from AC16 cells using TRIzol ${ }^{\circledR}$ reagent (Thermo Fisher Scientific, Inc.) according to the manufacturer's instructions. Total RNA was reverse transcribed into cDNA at $37^{\circ} \mathrm{C}$ for 15 min using 
PrimeScript RT Master Mix cDNA synthesis system (Bio-Rad Laboratories, Inc.). Subsequently, qPCR was performed using SYBR Green (Bio-Rad Laboratories, Inc.). The following thermocycling conditions were used for qPCR: Preheated at $95^{\circ} \mathrm{C}$ for $2 \mathrm{~min}$; followed by 34 cycles of $95^{\circ} \mathrm{C}$ for $10 \mathrm{sec}$, $60^{\circ} \mathrm{C}$ for $10 \mathrm{sec}$ and $72^{\circ} \mathrm{C}$ for $15 \mathrm{sec}$. The primers used for qPCR are presented in Table I. mRNA expression levels were quantified using the $2^{-\Delta \Delta C q}$ method (31) and normalized to the internal reference gene GAPDH.

In vitro cell viability assay. Cell viability was determined by performing a Cell Counting Kit-8 (CCK-8) assay (Beijing Solarbio Science \& Technology Co., Ltd.) according to the manufacturer's protocol. Briefly, AC16 cells were seeded into 96-well plates and grown to $75 \%$ confluence. Following transfection, CCK- 8 reagent was added to each well and incubated for $1 \mathrm{~h}$ at $37^{\circ} \mathrm{C}$. The absorbance of each well was measured at a wavelength of $450 \mathrm{~nm}$ on a microplate reader (Thermo Fisher Scientific, Inc.). For clonogenic assays, AC16 cells were plated into 6-well plates at a density of 4,000-8,000 cells/well. After $24 \mathrm{~h}$ later, cells were cultured in a hypoxia chamber (Billups-Rothenberg, Inc.) at $37^{\circ} \mathrm{C}$ for the indicated time points. Cells were fixed with $10 \%$ formalin at room temperature for $10 \mathrm{~min}$, followed by staining with $0.02 \%$ crystal violet at room temperature for $10 \mathrm{~min}$. Cell numbers were counted under a dissection microscope.

Mitochondrial membrane potential analysis. Mitochondrial membrane potential was measured via flow cytometry using rhodamine-123 (Beijing Solarbio Science \& Technology Co., Ltd.). Following treatment, AC16 cells $\left(10^{5}\right.$ cells $\left./ \mathrm{ml}\right)$ were incubated with $5 \mu \mathrm{M}$ rhodamine -123 at $37^{\circ} \mathrm{C}$ for $30 \mathrm{~min}$. Cells were washed twice with PBS and re-suspended in $300 \mu \mathrm{l}$ PBS. Subsequently, cells were analyzed via FACSCanto II flow cytometer (BD Biosciences) and data analyzed with FlowJo v10 (FlowJo, LLC).

Transfection and immunoprecipitation. To knockdown hCINAP or HIF-1 $\alpha$ in AC16 cells, the following siRNAs (all purchased from Sangon Biotech Co., Ltd.) were used: siRNA-hCINAP (5'-GAGAGA AGGUGGAGUUAUU-3'), siRNA-HIF-1 $\alpha$ (5'-AAGCAUUUCUCUCAUUUCCUCAUGG-3') and siRNA-ctrl (scrambled siRNAs) (5'-GACUACUGGUCGUUG AACU-3'). AC16 cells (1x10 cells/well) were transfected with $30 \mathrm{nM}$ siRNA-ctrl, $30 \mathrm{nM}$ siRNA-hCINAP, $1 \mu \mathrm{g}$ empty vector or $1 \mu \mathrm{g}$ hCINAP-Flag vector at room temperature using Effectene Transfection Reagent (Qiagen $\mathrm{GmbH}$ ) according to the manufacturer's protocol and flow experiments were performed $48 \mathrm{~h}$ after transfection. To induce LDHA activation, AC16 cells were transfected with 50 or $100 \mathrm{ng}$ vector of hCINAP-Flag at room temperature using Effectene Transfection Reagent (Qiagen $\mathrm{GmbH}$ ) according to the manufacturer's protocol.

For co-immunoprecipitation experiments, total extracts of AC16 cells were lysed using IP lysis buffer $(150 \mathrm{mM}$ $\mathrm{NaCl}, 25 \mathrm{mM}$ Tris- $\mathrm{HCl}, \mathrm{pH}$ 7.4, 5\% glycerol, 1\% NP-40, $1 \mathrm{mM}$ EDTA). Lysate $(1 \mathrm{ml})$ was incubated overnight at $4{ }^{\circ} \mathrm{C}$ with $1 \mu \mathrm{g}$ hCINAP antibody. Protein A/G-Sepharose $(60 \mu \mathrm{l})$ was added to samples and incubated at room temperature for $4 \mathrm{~h}$ on a rotary shaker $(300 \mathrm{xg})$. The pellets were washed three times with $1 \mathrm{ml}$ lysis buffer and eluted by boiling with
$200 \mu 1$ SDS-PAGE loading buffer. Samples were analyzed via western blotting as described above.

Annexin V/PI staining. Cells were incubated with Annexin V-FITC Apoptosis Detection kit (Beijing Solarbio Science \& Technology Co., Ltd.) according to the manufacturer's protocol. Briefly, cells were stained with $5 \mu \mathrm{l}$ of Annexin V-FITC and $5 \mu \mathrm{l}$ PI at room temperature for $15 \mathrm{~min}$. Following centrifugation at $6,00 \mathrm{x} \mathrm{g}$ for $3 \mathrm{~min}$ at room temperature. The population of early apoptotic cells (Annexin $\mathrm{V}^{+} / \mathrm{PI}^{-}$) and late-apoptotic/necrotic cells $\left(\right.$ Annexin $\mathrm{V}^{+} / \mathrm{PI}^{+}$) was evaluated using a FACSCanto II flow cytometer (BD Biosciences) and both Annexin $\mathrm{V}^{+}$cell populations were considered to be apoptotic cells. Data was measured by FlowJo v10 (FlowJo, LLC).

Luciferase reporter assay. JASPAR (jaspar.genereg. net) was used to predict whether HIF-1 $\alpha$ bound to the promoter of hCINAP. Promoter regions were defined using Eukaryotic Promoter Database (epd.vital-it.ch). To construct pGL3-hCINAP promoter-luciferase constructs, hCINAP promoter $(-2350$ to $+50 \mathrm{bp})$ was amplified from genomic DNA and assembled into the pGL3-Basic vector (cat. no. E1751; Promega Corporation). To construct the truncated hCINAP promoter constructs, hCINAP-promoter-1 (-2350 to $-270 \mathrm{bp}$ ), hCINAP-promoter-2 (-901 to $+50 \mathrm{bp})$, hCINAP-promoter-3 (-901 to $-270 \mathrm{bp})$, hCINAP-promoter-4 (-901 to $-549 \mathrm{bp})$ and hCINAP-promoter-5 (-715 to $-270 \mathrm{bp})$ were amplified from the pGL3-hCINAP promoter-luciferase vector and assembled into the pGL3-Basic vector. The primers used for the establishment of promoter constructs are presented in Table I. AC16 cells were plated $\left(3 \times 10^{4}\right.$ cells/well) into 24-well plates and co-transfected with $150 \mathrm{ng}$ pGL3-hCINAP promoter-luciferase, $15 \mathrm{ng}$ pcDNA-HIF-1 $\alpha$ or empty vector and $15 \mathrm{ng}$ pRL-TK vector (cat. no. E2241; Promega Corporation) using Effectene Transfection Reagent (Qiagen $\mathrm{GmbH}$ ). At $36 \mathrm{~h}$ post-transfection, luciferase activities were measured using a Dual-luciferase reporter kit (Promega Corporation). Firefly luciferase activities were normalized to Renilla luciferase activities.

Intracellular immunostaining. AC16 cells $\left(2 \times 10^{5}\right)$ grown on glass coverslips were fixed with $4 \%$ paraformaldehyde at $4^{\circ} \mathrm{C}$ for $30 \mathrm{~min}$ and permeabilized with $0.5 \%$ Triton $\mathrm{X}-100$ for $15 \mathrm{~min}$ at room temperature. Subsequently, cells were blocked with $5 \%$ BSA at room temperature for $1 \mathrm{~h}$, followed by incubation with cytochrome $\mathrm{C}$ antibody (1:500) overnight at $4^{\circ} \mathrm{C}$. Following washing with PBS, cells were incubated with fluorescein-conjugated goat anti-mouse $\operatorname{IgG}(1: 1,000$; cat. no. A-21202; Thermo Fisher Scientific, Inc.) at room temperature for $1 \mathrm{~h}$. Subsequently, cells were counterstained at room temperature for 5 min with Hoechst 33258 (Beijing Solarbio Science \& Technology Co., Ltd.) and observed using an inverted fluorescence microscope (magnification, x200).

Statistical analysis. Data are presented the mean \pm SEM of at least three independent experiments. Comparisons among multiple groups were analyzed using one-way ANOVA followed by Tukey's post hoc test. Statistical analyses were performed using SPSS software (version 22.0; IBM Corp.). $\mathrm{P}<0.05$ was considered to indicate a statistically significant difference. 
A
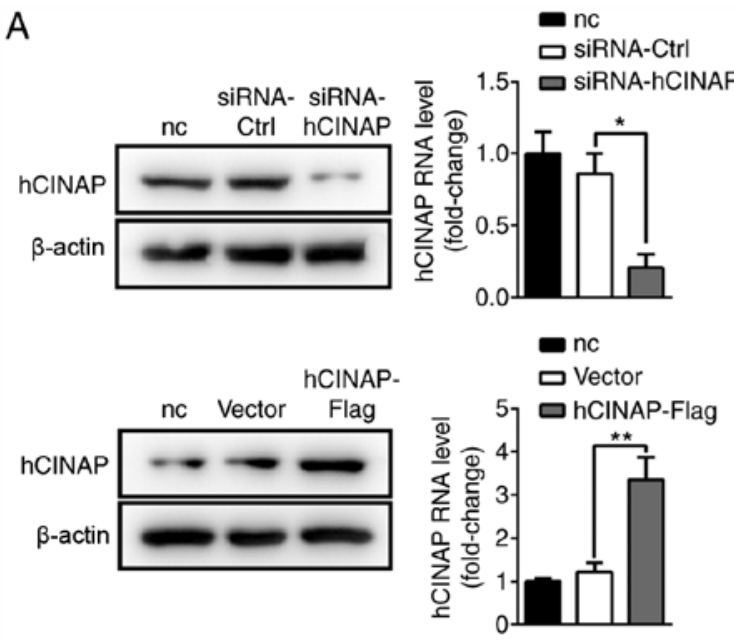

hCINAP.

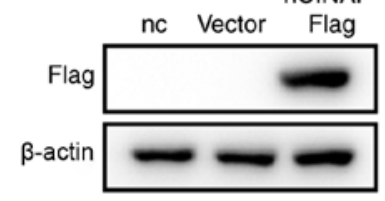

B Hypoxia 0
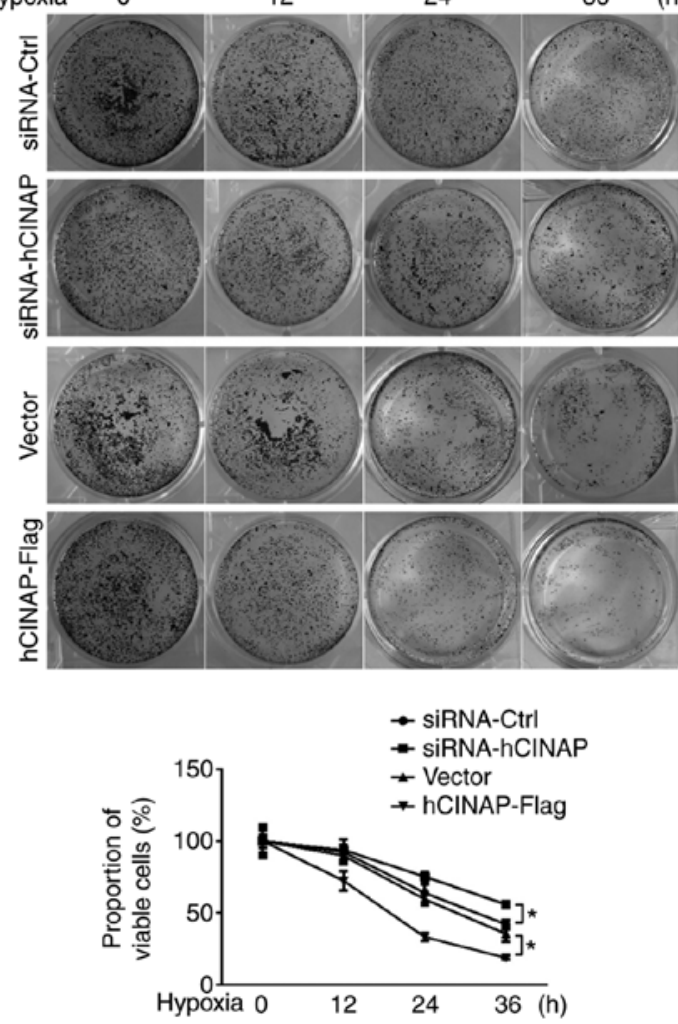

C
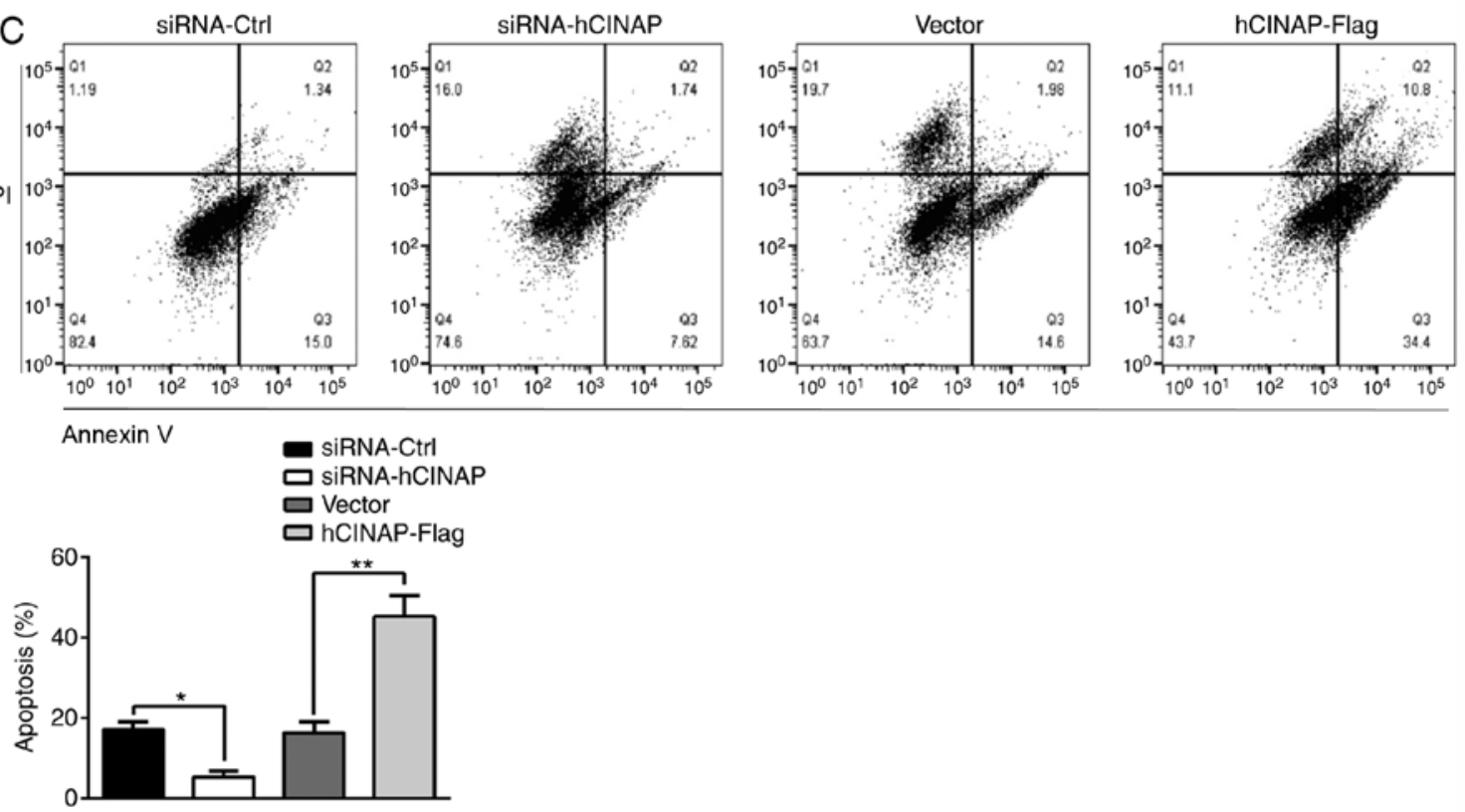

Hypoxia

Figure 1. hCINAP knockdown prevents hypoxia-induced AC16 cell apoptosis. AC16 cells were transfected with siRNA-Ctrl, siRNA-hCINAP, vector or hCINAP-Flag. (A) Western blotting was performed to determine the expression levels of hCINAP and Flag. (B) Cells were cultured under hypoxic conditions for $0,12,24$ or $36 \mathrm{~h}$, and then cell Counting Kit-8 and clonogenic assays were performed to assess AC16 cell viability. (C) Cells were cultured under hypoxic conditions for $12 \mathrm{~h}$ and then AC16 cell apoptosis was assessed via Annexin V/PI staining followed by flow cytometry. ${ }^{*} \mathrm{P}<0.05$ and ${ }^{* *} \mathrm{P}<0.01$. hCINAP, human coilin interacting nuclear ATPase protein; siRNA, small interfering RNA; Ctrl, control; nc, negative control.

\section{Results}

hCINAP knockdown increases cell survival in response to hypoxia. To identify the role of hCINAP in hypoxia, gainand loss-of-function experiments were performed using hCINAP-Flag vector and siRNA-hCINAP, respectively.
Compared with siRNA-Ctrl, siRNA-hCINAP decreased hCINAP mRNA and protein expression levels, whereas compared with vector, hCINAP-Flag markedly elevated hCINAP expression levels (Fig. 1A). Subsequently, whether altered hCINAP expression affected AC16 cell viability under hypoxia was investigated. The results indicated that $\mathrm{AC} 16$ cell 
A

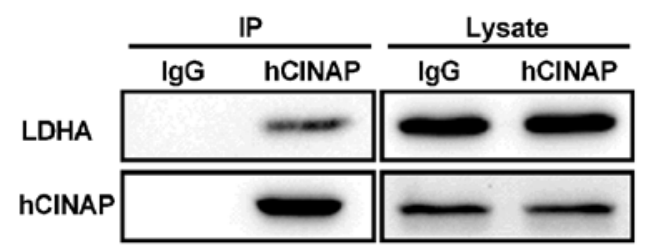

C
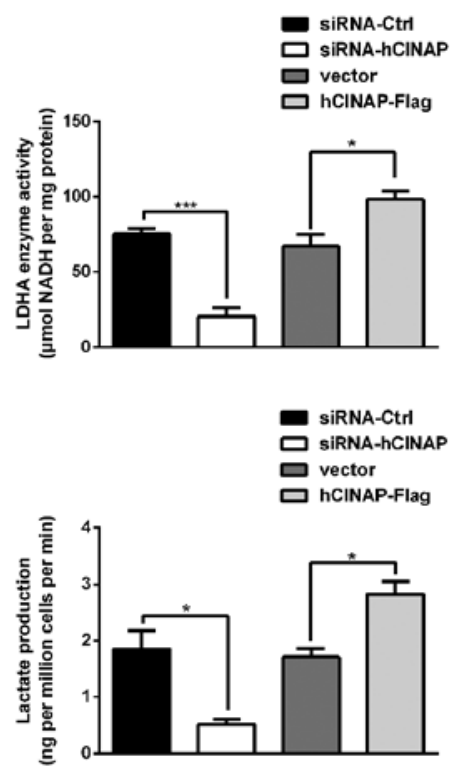

\section{$\mathrm{E}$}

B

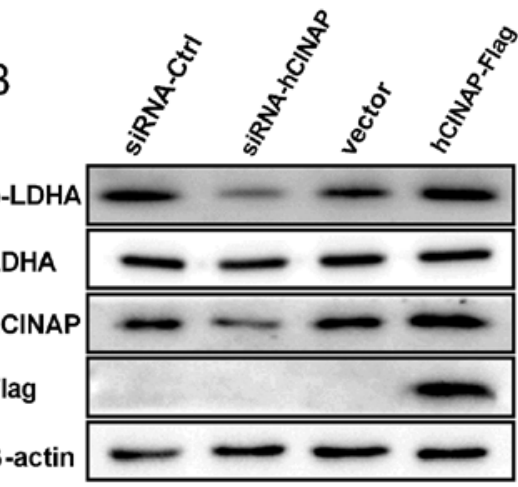

D

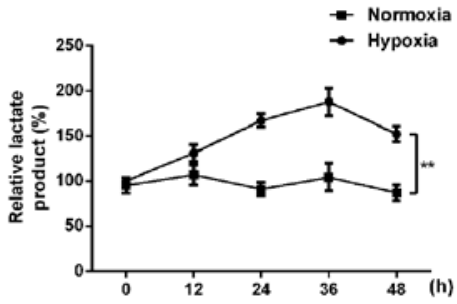

vector
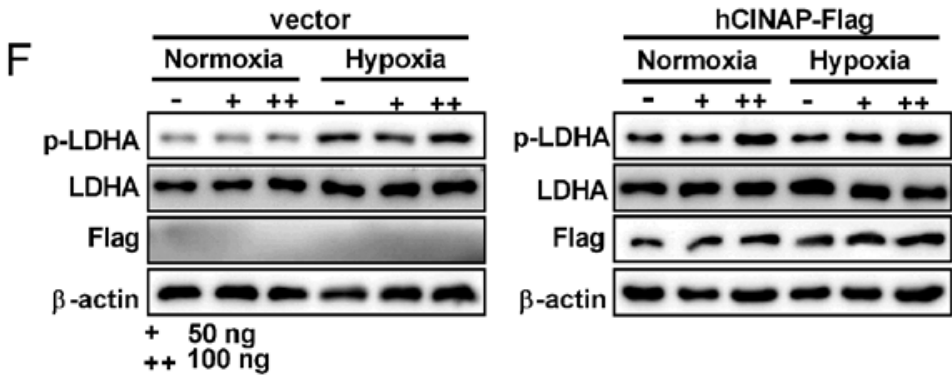

Figure 2. hCINAP facilitates LDHA phosphorylation and lactate production. (A) AC16 cells were transfected with or without hCINAP-Flag. Subsequently, immunoprecipitation was performed using anti-hCINAP and then western blotting was performed. (B) AC16 cells were transfected with siRNA-Ctrl, siRNA-hCINAP, vector or hCINAP-Flag. Subsequently, western blotting was performed to measure p-LDHA, LDHA, hCINAP and Flag protein expression levels. (C) LDHA enzyme activities. Lactate levels in AC16 cells (D) under normoxic and hypoxic conditions or (E) following transfection with siRNA-Ctrl, siRNA-hCINAP, vector or hCINAP-Flag. (F) AC16 cells were transfected with 50 or $100 \mathrm{ng}$ vector of hCINAP-Flag. Subsequently western blotting was performed to measure p-LDHA, LDHA and Flag protein expression levels under normoxic and hypoxic conditions. ${ }^{*} \mathrm{P}<0.05,{ }^{* *} \mathrm{P}<0.01$ and ${ }^{* * * *} \mathrm{P}<0.001$. hCINAP, human coilin interacting nuclear ATPase protein; LDHA, lactate dehydrogenase A; siRNA, small interfering RNA; Ctrl, control; p, phosphorylated; IP, immunoprecipitation.

viability of control group (siRNA-ctrl group and vector group) was gradually decreased by $\sim 50 \%$ over the $36 \mathrm{~h}$ of hypoxia. Following hypoxia for $36 \mathrm{~h}$, cell viability was significantly higher in hCINAP-knockdown cells compared with the siRNA-Ctrl group, whereas cell viability was significantly decreased in hCINAP-overexpression cells compared with the vector group. Similar results were obtained for the clonogenic assay (Fig. 1B). The flow cytometry results indicated that the percentage of apoptotic cells in the siRNA-hCINAP group was significantly decreased compared with the siRNA-Ctrl group, whereas hCINAP overexpression displayed the opposite effect compared with the vector group (Fig. 1C), indicating that hCINAP knockdown inhibited apoptosis under hypoxic conditions. Collectively, the aforementioned results suggested that hCINAP knockdown promoted AC16 cell survival.

hCINAP overexpression elevates hypoxia-induced lactate production via facilitating LDHA phosphorylation. It has been reported that hCINAP binds to LDHA and promotes the phosphorylation of LDHA in colorectal cancer stem cells (25). 
Therefore, it was hypothesized that hCINAP interacted with LDHA in AC16 cells and co-immunoprecipitation assays were performed. The results indicated that exogenous hCINAP interacted with LDHA in AC16 cells (Fig. 2A). Compared with the siRNA-Ctrl group, hCINAP knockdown markedly decreased LDHA phosphorylation, whereas compared with the vector group, hCINAP overexpression notably increased LDHA phosphorylation (Fig. 2B). Alterations to hCINAP expression displayed no obvious effects on LDHA expression, which was consistent with a previous study (29). Accordingly, LDHA activity was significantly decreased by hCINAP knockdown compared with the siRNA-Ctrl group. By contrast, hCINAP overexpression significantly increased LDHA activity compared with the vector group (Fig. 2C). Lactate serves as an energy substrate and gluconeogenic precursor, which is essential for cardiomyocyte survival under hypoxic conditions (32). In the present study, lactate accumulation was increased in AC16 cells following hypoxia exposure for $12 \mathrm{~h}$ compared with the normoxia group, but lactate accumulation in the hypoxia and normoxia groups began to fall at $36 \mathrm{~h}$ (Fig. 2D). In AC16 cells, hCINAP knockdown significantly decreased lactate production compared with the siRNA-Ctrl group, whereas hCINAP overexpression significantly increased lactate production compared with the vector group (Fig. 2E). Furthermore, compared with the vector + hypoxia group, hCINAP overexpression markedly increased hypoxia-induced LDHA phosphorylation in a dose-dependent manner (Fig. 2F). The results indicated that hCINAP promoted hypoxia-induced lactate production via binding to LDHA and modulating LDHA activity.

$H I F-\alpha$ is recruited to the hCINAP promoter in hypoxia. To further investigate the role of hCINAP in hypoxia, whether hCINAP protein expression was increased under hypoxia was investigated. HIF-1 $\alpha$ expression gradually increased in a time-dependent manner in response to hypoxia. Similarly, hCINAP protein expression was also increased in a time-dependent manner in response to hypoxia (Fig. 3A). To assess whether HIF-1 $\alpha$ regulated hypoxia-induced hCINAP, AC16 cells were transfected with siRNAs targeted against HIF-1 $\alpha$. The western blotting results demonstrated that hCINAP protein expression levels were markedly reduced in the siRNA-HIF-1 $\alpha$ group compared with the siRNA-Ctrl group under normoxic and hypoxic conditions (Fig. 3B), suggesting that HIF-1 $\alpha$ expression was required for hCINAP expression. The appropriate hCINAP promoter sequence ranging from $-2,350 \mathrm{bp}$ upstream to $+50 \mathrm{bp}$ downstream of the transcription start site was retrieved from the Eukaryotic Promoter Database (epd.vital-it.ch). Subsequently, the hCINAP promoter was screened for the presence of HIF-1 $\alpha$ binding sites using JASPAR (jaspar.genereg.net). Luciferase reporter assays were performed to identify HIF- $1 \alpha$ binding sites within the hCINAP promoter. Compared with the vector $+\mathrm{HIF}-1 \alpha$ group, HIF-1 $\alpha$ overexpression significantly increased the luciferase activity of the hCINAP promoter $(-2,350$ to $+50 \mathrm{bp})$, suggesting that HIF-1 $\alpha$ bound to the hCINAP promoter and promoted its transcription. Subsequently, two different regions of the hCINAP promoter were designed (hCINAP-promoter-1, $-2,350$ to $-270 \mathrm{bp}$; hCINAP-promoter- $2,-901$ to $+50 \mathrm{bp}$ ) and analyzed by performing luciferase reporter assays. Compared with the vector + HIF-1 $\alpha$ group, HIF-1 $\alpha$ overexpression significantly increased the luciferase activity of hCINAP-promoter-1 and hCINAP-promoter-2, indicating that the overlap region of the two promoter regions was required for HIF-1 $\alpha$ binding. Subsequently, the overlap region (-901 to $-270 \mathrm{bp}$ ) with the hCINAP promoter was constructed as hCINAP-promoter- 3 and further segmented into hCINAP-promoter-4 (-901 to -549 bp) and hCINAP-promoter-5 (-715 to $-270 \mathrm{bp})$. Compared with the vector + HIF-1 $\alpha$ group, HIF-1 $\alpha$ overexpression significantly enhanced the luciferase activity of hCINAP-promoter-3 and hCINAP-promoter-4, but failed to significantly alter the activity of hCINAP-promoter-5, suggesting that the specific region (-901 to $-715 \mathrm{bp}$ ) of hCINAP-promoter-4 was required for HIF-1 $\alpha$ binding (Fig. 3C). Furthermore, this key region of the hCINAP promoter for HIF-1 $\alpha$ binding was confirmed by performing a ChIP-qPCR assay under hypoxic conditions (Fig. 3D). Collectively, the results demonstrated that hCINAP was a direct target of HIF-1 $\alpha$.

hCINAP knockdown attenuates hypoxia-induced apoptosis via the mitochondrial-mediated signaling pathway. Emerging evidence has indicated that hypoxia-induced apoptosis involving activation of Caspases can occur in a cytochrome C-dependent manner (27). Subsequently, the role of hCINAP in cytochrome $\mathrm{C}$ release and Caspase activation was examined. Immunofluorescence staining indicated that under hypoxic conditions, cytochrome $\mathrm{C}$ was released into the cytoplasm in siRNA-Ctrl-transfected cells, whereas hCINAP knockdown inhibited cytochrome C (Fig. 4A). Consistently, the western blotting results indicated that hypoxia led to cytochrome $\mathrm{C}$ release from the mitochondria into the cytoplasm in siRNA-Ctrl-transfected cells, but this effect was not observed in hCINAP-knockdown cells (Fig. 4B), suggesting that hCINAP was involved in the mitochondrial-mediated apoptotic signaling pathway. Furthermore, compared with the siRNA-Ctrl group, hCINAP knockdown significantly decreased hypoxia-induced activation of Caspase-3 and -9 , whereas hCINAP overexpression displayed the opposite effect on Caspase activation compared with the vector group. Moreover, hCINAP displayed no significant effect on Caspase activation under normoxic conditions (Fig. 4C). In addition, compared with the hypoxia + siRNA-Ctrl group, hCINAP knockdown significantly inhibited hypoxia-induced depletion of the mitochondrial membrane potential (Fig. 4D). Therefore, the results indicated that hCINAP knockdown protected AC16 cells against hypoxia-induced apoptosis via the mitochondrial-mediated apoptotic signaling pathway.

hCINAP knockdown inhibits lactate-induced cardiomyocyte apoptosis. The aforementioned results indicated that hCINAP promoted LDHA phosphorylation and hypoxia-induced apoptosis. Therefore, whether hCINAP was involved in lactate accumulation-induced cell damage was investigated. Following treatment with $20 \mathrm{mM}$ lactate for $24 \mathrm{~h}$, AC16 cells displayed significantly increased apoptosis compared with control cells, which was significantly reduced by transfection with siRNA-hCINAP compared with siRNA-Ctrl (Fig. 5A), indicating that hCINAP knockdown blocked lactate-induced apoptosis. To assess the role of hCINAP in the regulation of lactate-induced apoptosis via LDHA, an LDHA inhibitor, 

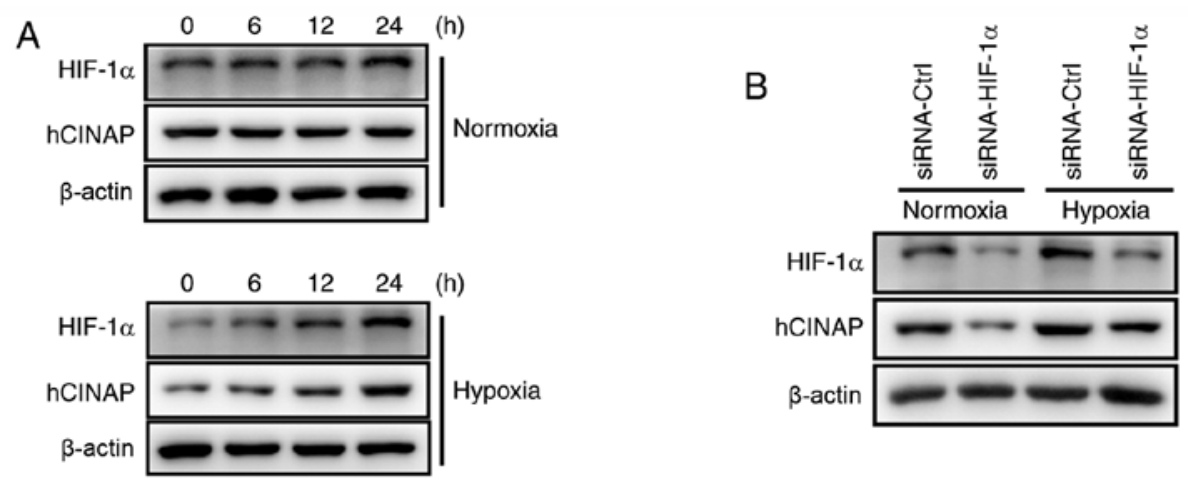

C
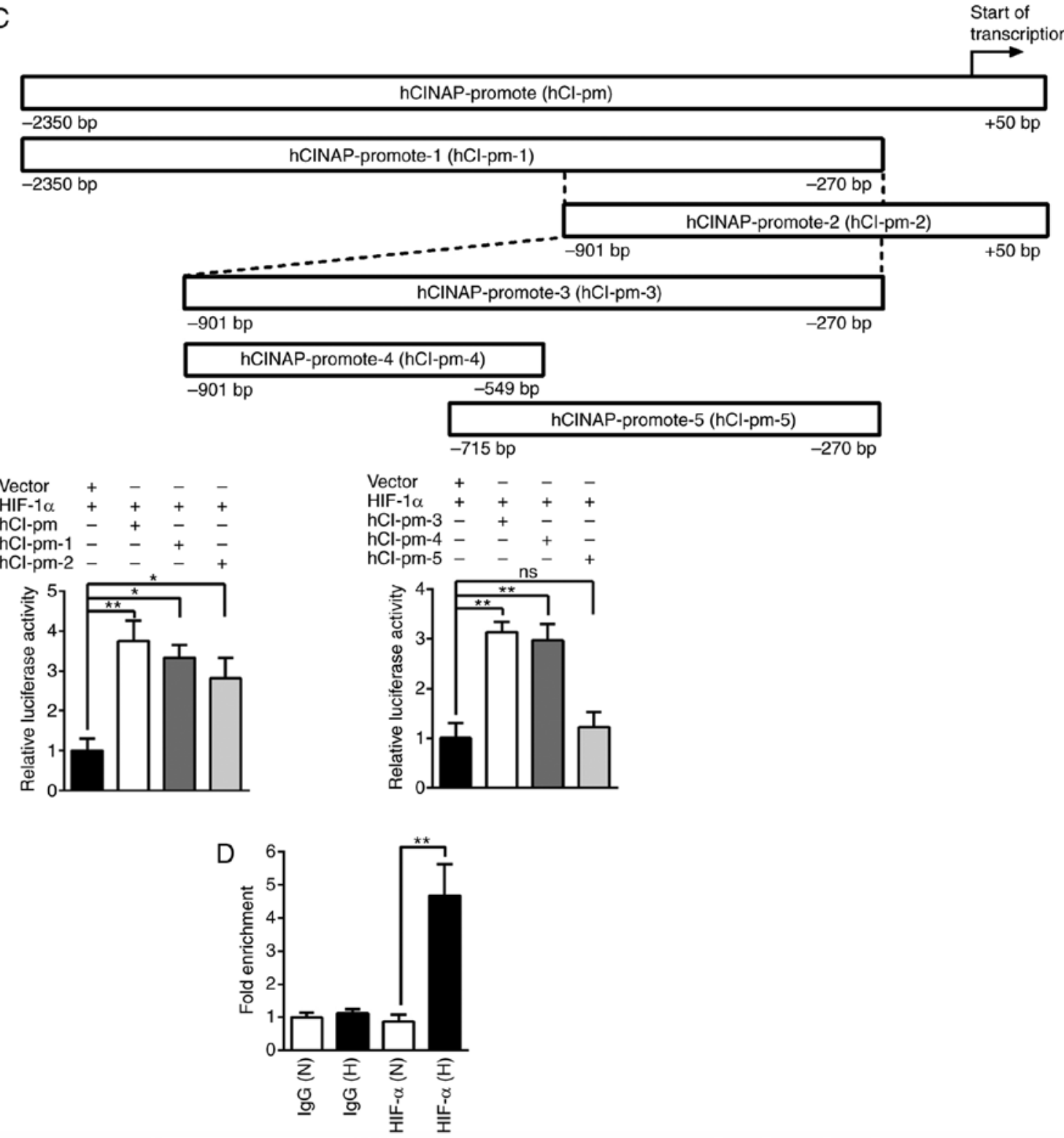

Figure 3. HIF-1 $\alpha$ promotes hCINAP expression by directly binding to the hCINAP promoter. (A) Western blotting was performed to measure HIF-1 $\alpha$ and hCINAP protein expression levels in AC16 cells under normoxic or hypoxic conditions. (B) Following transfection with siRNA-Ctrl or siRNA-HIF-1 $\alpha$, western blotting was performed to measure HIF-1 $\alpha$ and hCINAP protein expression levels in AC16 cells under normoxic or hypoxic conditions. (C) Different regions of the hCINAP promoter (hCI-pm-1, -2,-3 -4 and -5) were constructed into a luciferase reporter vector, and luciferase reporter assays were performed to determine the effects of HIF-1 $\alpha$ on different partial regions of hCINAP promoter activity. (D) Chromatin-immunoprecipitation-quantitative PCR was performed to verify hCINAP as a direct binding target of HIF- $1 \alpha$. ${ }^{*} \mathrm{P}<0.05$ and ${ }^{* * *} \mathrm{P}<0.01$. HIF-1 $\alpha$, hypoxia-inducible factor-1 $\alpha$; hCINAP, human coilin interacting nuclear ATPase protein; siRNA, small interfering RNA; Ctrl, control; ns, not significant; N, normoxia; H, hypoxia.

FX11, was used to downregulate LDHA activity. Compared with the vector group, hCINAP overexpression markedly increased Caspase- 3 and -9 activation in response to lactate treatment (Fig. 5B). However, LDHA inhibition by FX11 

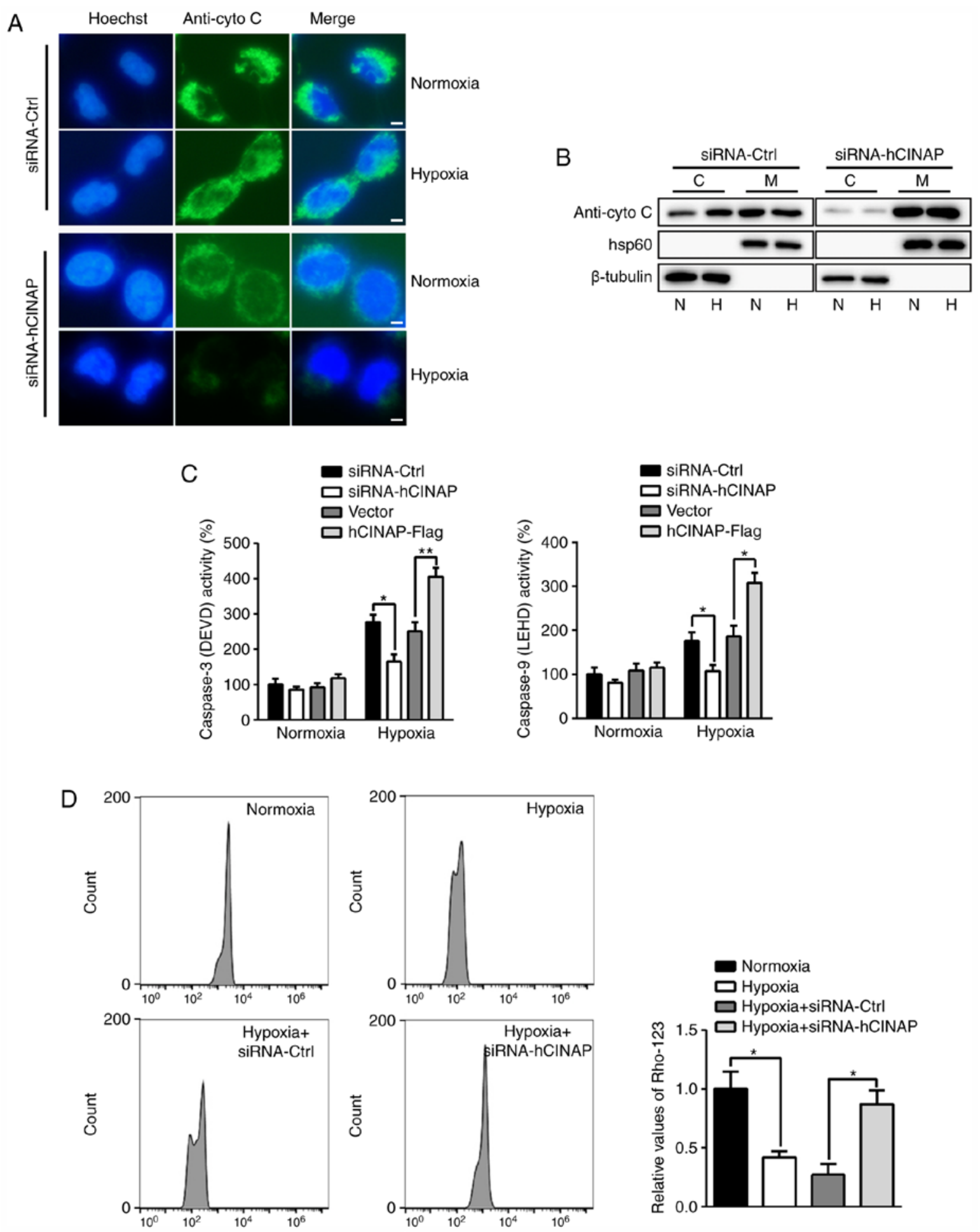

Figure 4. hCINAP regulates mitochondrial-mediated apoptosis in hypoxia. Following transfection with siRNA-Ctrl, siRNA-hCINAP, vector or hCINAP-Flag, AC16 cells were exposed to normoxia or hypoxia. (A) Cells were harvested and stained with anti-cytochrome C and Hoechst 33258. Scar bar, $20 \mu \mathrm{m}$. (B) Western blotting was performed to measure the protein expression levels of cytochrome $\mathrm{C}$ in cytosolic and mitochondrial fractions. (C) Caspase activities were measured using luminogenic substrate of Ac-DEVD-AMC (Caspase-3) and Z-LEHD-aminoluciferin (Caspase-9). (D) Mitochondrial membrane potentials were assessed by staining with rhodamine- 123 followed by flow cytometry. ${ }^{*} \mathrm{P}<0.05$ and ${ }^{* *} \mathrm{P}<0.01$. hCINAP, human coilin interacting nuclear ATPase protein; siRNA, small interfering RNA; Ctrl, control; C, cytosolic; M, mitochondrial; N, normoxia; H, hypoxia; Rho-123, rhodamine-123; cyto C, cytochrome C.

significantly attenuated the effect of hCINAP overexpression on Caspase-3 and -9 activation, suggesting that hCINAP regulated Caspase activation via LDHA. Furthermore, blocking of LDHA significantly decreased hCINAP overexpression-induced excessive Caspase- 3 and -9 activation under hypoxic conditions (Fig. 5C). In addition, compared with the corresponding control groups, hCINAP knockdown and overexpression displayed no notable effects on MCT1 expression, which facilitates lactate entry into or efflux out of cells depending on their metabolic state (33) (Fig. 5D). Collectively, the results suggested that hCINAP knockdown mitigated lactate accumulation-induced apoptosis. 
A
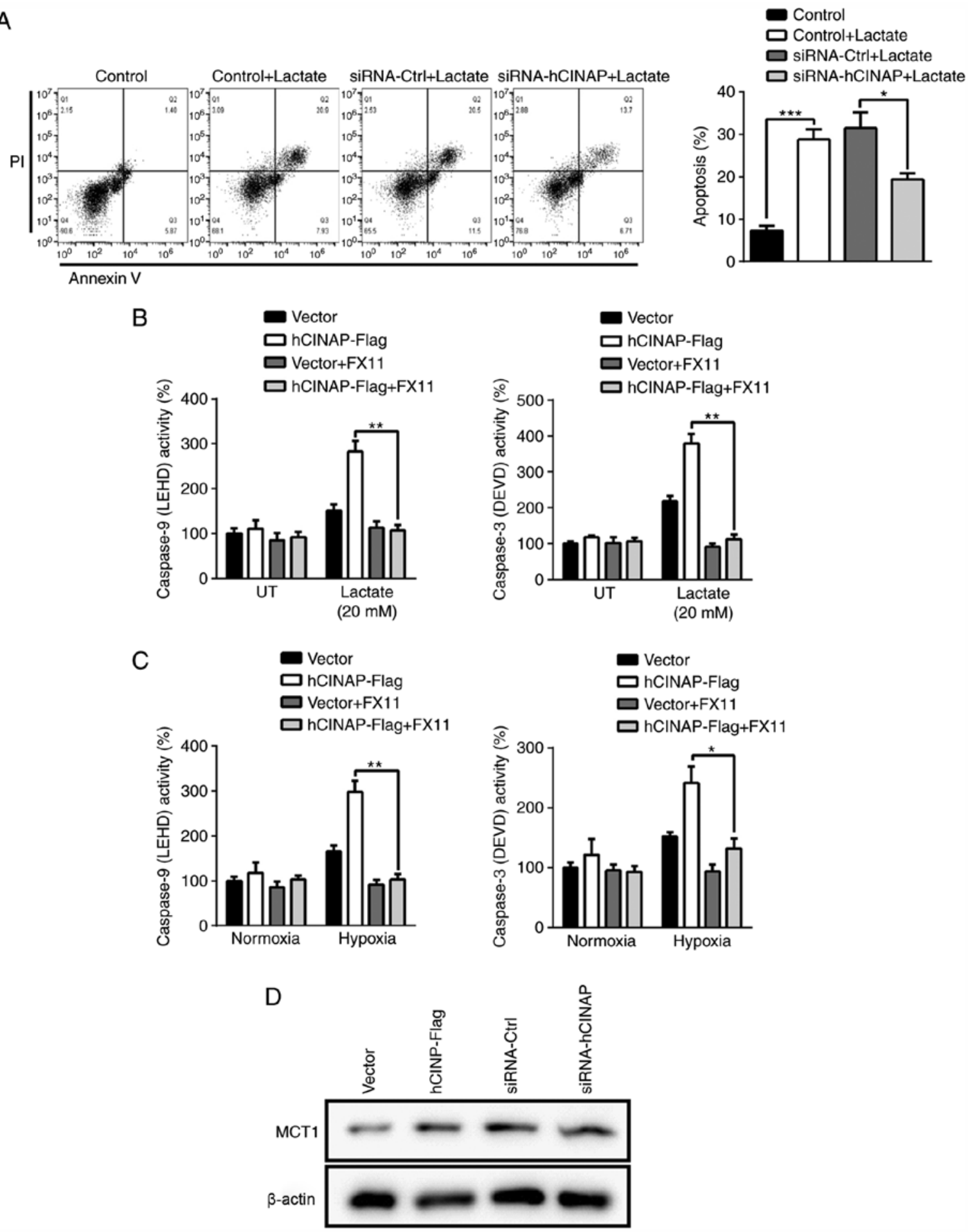

Figure 5. hCINAP knockdown prevents lactate-induced apoptosis. (A) Following transfection with siRNA-Ctrl or siRNA-hCINAP, AC16 cells were treated with lactate for $12 \mathrm{~h}$. Cell apoptosis was examined by performing Annexin V/PI staining followed by flow cytometry. (B) Following transfection with vector or hCINAP-Flag, AC16 cells were treated with or without LDHA inhibitor FX11 $(10 \mu \mathrm{M})$, then treated with or without lactate $(20 \mathrm{mM})$ for $12 \mathrm{~h}$. Caspase activities were measured using luminogenic substrate of Ac-DEVD-AMC (Caspase-3) and Z-LEHD-aminoluciferin (Caspase-9). (C) Following transfection with vector or hCINAP-Flag, AC16 cells were treated with or without LDHA inhibitor FX11 (10 $\mu \mathrm{M})$, then exposed to normoxia or hypoxia for $24 \mathrm{~h}$. Caspase activities were measured using luminogenic substrate of Ac-DEVD-AMC (Caspase-3) and Z-LEHD-aminoluciferin (Caspase-9). (D) Following transfection with siRNA-Ctrl, siRNA-hCINAP, vector or hCINAP-Flag, western blotting was performed to measure MCT1 protein expression levels in AC16 cells ${ }^{*} \mathrm{P}<0.05,{ }^{* *} \mathrm{P}<0.01$ and ${ }^{* * *} \mathrm{P}<0.001$. hCINAP, human coilin interacting nuclear ATPase protein; siRNA, small interfering RNA; Ctrl, control; LDHA, lactate dehydrogenase A; MCT1, monocarboxylate transporter 1; UT, untreated.

\section{Discussion}

Oxygen deprivation of cardiomyocytes causes a notable alteration in gene expression and characterizes major pathological processes, such as myocardial ischemia and myocardial infarction (3). Cardiomyocyte apoptosis is a major cellular injury that occurs during AMI (34). Therefore, understanding the potential molecular mechanisms underlying hypoxia-induced 
apoptosis could aid with the development of therapeutic strategies to prevent and treat AMI. In the present study, the results indicated that hCINAP mediated lactate production via LDHA. Compared with normoxia conditions, hCINAP expression was increased under hypoxic conditions, leading to LDHA activation. hCINAP knockdown attenuated mitochondrial-mediated apoptosis signaling in response to hypoxia.

Homozygous hCINAP knockout mice display embryonic lethality as ATPase hCINAP regulates $18 \mathrm{~S}$ rRNA processing, and is essential for embryogenesis and tumor growth (35). However, the specific role of hCINAP in cardiomyocytes is not completely understood. The results of the present study indicated that compared with the vector group, hCINAP overexpression decreased cardiomyocyte viability, and compared with the siRNA-Ctrl group, hCINAP knockdown reduced apoptosis under hypoxic conditions. Conversely, a previous study reported that hCINAP knockdown increased apoptosis in colorectal cancer stem cells, whereas colon organoids displayed low sensitivity to hCINAP knockdown-induced alterations to apoptosis (29), indicating that hCINAP serves different roles in cancer cells compared with healthy cells. Cancer cells can acquire necessary nutrients from the malnourished environment, which are then used to maintain viability and build new biomass (36). A major characteristic of tumor cells is the generation of ATP via glycolysis followed by lactate production, instead of oxidative phosphorylation, even when sufficient oxygen is present, a phenomenon that is known as the Warburg effect or aerobic glycolysis (37). It has been suggested that hCINAP enhanced aerobic glycolysis via the positive regulation of LDHA activity, leading to the generation of extracellular lactate to provide a favorable microenvironment for CRCSC proliferation and invasion (29). In the present study, the results demonstrated that hCINAP interacted with LDHA, and hCINAP was required for the phosphorylation of LDHA in cardiomyocytes. Furthermore, compared with the siRNA-Ctrl group, hCINAP knockdown impaired LDHA activity, suggesting that LDHA phosphorylation was responsible for its enzymatic activity. Lactate is formed by a lack of oxygen and is utilized under fully aerobic conditions in mitochondria. The anaerobic metabolism causes overproduction of lactate and metabolic acidosis, which results in cardiomyocyte damage (38). Consistent with previous reports, the present study demonstrated that cardiomyocytes exposed to hypoxia for a short duration displayed increased lactate production, whereas hypoxia exposure for a long duration decreased lactate levels $(18,19)$. Moreover, compared with the siRNA-Ctrl group, hCINAP knockdown suppressed hypoxia-induced lactate production. Collectively, the aforementioned results suggested that hCINAP knockdown protected against hypoxia-induced damage by regulating the lactate pathway.

Transcriptional induction of LDHA is caused by hypoxia, with both HIF-1 $\alpha$ and HIF-2 $\alpha$ binding to LDHA at 89 bp under hypoxic conditions (23). In the present study, HIF-1 $\alpha$ bound 901-715 bp upstream of the hCINAP transcription start site, suggesting that hypoxia-induced expression of hCINAP was dependent on HIF-1 $\alpha$. Alterations to hCINAP expression levels did not affect the total expression levels of LDHA, indicating that hCINAP was involved in the post-transcriptional regulation of LDHA.
Under normal conditions, ROS display beneficial effects as they regulate several important physiological reactions via redox-responsive signaling pathways (39). Additionally, ROS regulate cellular proliferation, differentiation, vascular tone and adhesion (40). Tissue hypoxia elevates intracellular ROS levels, leading to deregulation of cellular ion homeostasis, cell death and tissue inflammation (10). Moreover, Bcl-2 has been reported to modulate antioxidant defenses and lipid peroxidation without altering intracellular ROS levels (41).

Lactate accumulation-induced cytosolic acidification leads to mitochondrial-dependent apoptosis, including dissipation of mitochondrial membrane potential, release of cytochrome $\mathrm{C}$ and activation of Caspases (42). The results of the present study suggested that hCINAP promoted hypoxia-induced apoptosis by increasing cytochrome $\mathrm{C}$ release. In addition, compared with the siRNA-Ctrl group, hCINAP knockdown attenuated hypoxia-induced mitochondrial membrane potential dissipation and impaired the activity of Caspase- 3 and -9 , indicating that hCINAP aggravated hypoxia-induced cell death involving Caspases. However, alterations to hCINAP expression did not significantly affect Caspase activity under normoxic conditions. It is likely that low levels of lactate are not sufficient for inducing Caspase activity under normoxic conditions. Therefore, the results indicated that hCINAP may participate in the regulation of mitochondrial-mediated apoptosis under hypoxic conditions. A previous study also emphasized the oncogene function of hCINAP in acute myeloid leukemia model mice (28). hCINAP knockdown results in higher levels of DNA damage and renders cells more sensitive to chemotherapeutic drugs (28). MCT1 is abundantly expressed in heart tissue localized in mitochondrial membranes, where it facilitates transport of lactate into mitochondria for oxidative metabolism (33). Under pathological conditions, such as hypoxia and ischemia, increased MCT1 expression mitigates intracellular acidification (19). In the right atrial appendage of patients with atrial fibrillation, cytoplasmic lactate concentration and mitochondrial MCT1 expression were elevated, which suggested that highly expressed MCT1 is closely related to cardiovascular diseases (43). Previous study reported that MCT1 protein expression was significantly increased in lactate-stimulated cardiomyocytes when compared with unstimulated cells (44), whereas hCINAP knockdown did not notably affect MCT1 expression levels compared with the siRNA-Ctrl group, indicating that hCINAP regulated lactate signaling via specifically binding to LDHA. Furthermore, hCINAP serves as a negative regulator in $\mathrm{NF}-\kappa \mathrm{B}$ signaling by recruiting serine/threonine-protein phosphatase 1 to deactivate IKK (45); therefore, the role of hCINAP in hypoxia-induced inflammatory signaling requires further investigation.

Collectively, the results of the present study demonstrated that hCINAP-knockdown cardiomyocytes displayed higher cell viability and lower apoptosis compared with the siRNA-Ctrl group, and hCINAP expression was increased in a HIF- $1 \alpha$-dependent manner under hypoxic conditions. The results also indicated that hCINAP was involved in mitochondrial-mediated apoptosis, including the release of cytochrome $\mathrm{C}$ and activation of Caspases. Furthermore, compared with the siRNA-Ctrl group, hCINAP knockdown suppressed lactate accumulation via inhibiting LDHA activity, which might contribute to the protective effects of hCINAP in 
hypoxia-induced cardiomyocytes. Based on the important role of hCINAP in regulating hypoxia-induced cell death, understanding the transcriptional network that regulates hCINAP expression will aid with the identification of genes that regulate hCINAP expression under hypoxic conditions. Unfortunately, at present, there are no drugs or inhibitors that specifically target hCINAP, although it is a promising candidate for cancer treatment (46). The protective effect of hCINAP knockdown against cardiomyocyte apoptosis identified in the present study suggested that selective small-molecule inhibitors and monoclonal antibodies targeting hCINAP may serve as therapeutic strategies for hypoxia-related diseases, such as chronic obstructive pulmonary disease, chronic mountain sickness and tumorigenesis.

\section{Acknowledgements}

Not applicable.

\section{Funding}

The present study was supported by the National Nature Science Foundation of China (grant no. 81470247).

\section{Availability of data and materials}

The datasets used and/or analyzed during the current study are available from the corresponding author on reasonable request.

\section{Authors' contributions}

HX and GX designed the experiments. HX,ZY and GX analyzed the data. ZY and YG wrote the manuscript. All authors contributed to data interpretation and critically revised the manuscript. All authors read and approved the final manuscript.

\section{Ethics approval and consent to participate}

Not applicable.

\section{Patient consent for publication}

Not applicable.

\section{Competing interests}

The authors declare that they have no competing interests.

\section{References}

1. Benjamin EJ, Virani SS, Callaway CW, Chamberlain AM, Chang AR, Cheng S, Chiuve SE, Cushman M, Delling FN, Deo R, et al: Heart disease and stroke statistics-2018 update: A report from the American heart association. Circulation 137: e67-e492, 2018.

2. Halestrap AP and Wilson MC: The monocarboxylate transporter family-role and regulation. IUBMB Life 64: 109-119, 2012.

3. Anderson JL and Morrow DA: Acute myocardial infarction. N Engl J Med 376: 2053-2064, 2017.

4. Nakada Y, Canseco DC, Thet S, Abdisalaam S, Asaithamby A, Santos CX, Shah AM, Zhang H, Faber JE, Kinter MT, et al Hypoxia induces heart regeneration in adult mice. Nature 541: 222-227, 2017
5. Yin J, Ni B, Liao WG and Gao YQ: Hypoxia-induced apoptosis of mouse spermatocytes is mediated by HIF-1 $\alpha$ through a death receptor pathway and a mitochondrial pathway. J Cell Physiol 233: 1146-1155, 2018

6. Zhang H, Liu B, Li T, Zhu Y, Luo G, Jiang Y, Tang F, Jian Z and Xiao Y: AMPK activation serves a critical role in mitochondria quality control via modulating mitophagy in the heart under chronic hypoxia. Int J Mol Med 41: 69-76, 2018.

7. Kim R, Emi M and Tanabe K: Role of mitochondria as the gardens of cell death. Cancer Chemother Pharmacol 57: 545-553, 2006.

8. Balaban RS, Nemoto S and Finkel T: Mitochondria, oxidants, and aging. Cell 120: 483-495, 2005.

9. Soltani M, Moghimian M, Abtahi-Eivari SH, Shoorei H, Khaki A and Shokoohi M: Protective effects of matricaria chamomilla extract on torsion/detorsion-induced tissue damage and oxidative stress in adult rat testis. Int J Fertil Steril 12: 242-248, 2018.

10. Lokmic Z, Musyoka J, Hewitson TD and Darby IA: Hypoxia and hypoxia signaling in tissue repair and fibrosis. Int Rev Cell Mol Biol 296: 139-185, 2012.

11. Shokoohi M, Khaki A, Shoorei H, Khaki AA, Moghimian M and Abtahi-Eivary SH: Hesperidin attenuated apoptotic-related genes in testicle of a male rat model of varicocoele. Andrology 8: 249-258, 2020.

12. Ameli M, Hashemi MS, Moghimian M and Shokoohi M: Protective effect of tadalafil and verapamil on testicular function and oxidative stress after torsion/detorsion in adult male rat. Andrologia 50: e13068, 2018

13. Denko NC: Hypoxia, HIF1 and glucose metabolism in the solid tumour. Nat Rev Cancer 8: 705-713, 2008.

14. Mandl M and Depping R: Hypoxia-inducible aryl hydrocarbon receptor nuclear translocator (ARNT) (HIF-1 $\beta$ ): Is it a rare exception? Mol Med 20: 215-220, 2014.

15. Depping R, Jelkmann W and Kosyna FK: Nuclear-cytoplasmatic shuttling of proteins in control of cellular oxygen sensing. J Mol Med (Berl) 93: 599-608, 2015.

16. Brahimi-Horn MC, Bellot $\mathrm{G}$ and Pouyssegur J: Hypoxia and energetic tumour metabolism. Curr Opin Genet Dev 21: 67-72, 2011.

17. Loor G and Schumacker PT: Role of hypoxia-inducible factor in cell survival during myocardial ischemia-reperfusion. Cell Death Differ 15: 686-690, 2008.

18. Evans RK, Schwartz DD and Gladden LB: Effect of myocardial volume overload and heart failure on lactate transport into isolated cardiac myocytes. J Appl Physiol (1985) 94: 1169-1176, 2003.

19. Johannsson E, Lunde PK, Heddle C, Sjaastad I, Thomas MJ, Bergersen L, Halestrap AP, Blackstad TW, Ottersen OP and Sejersted OM: Upregulation of the cardiac monocarboxylate transporter MCT1 in a rat model of congestive heart failure. Circulation 104: 729-734, 2001.

20. Fiume L, Manerba M, Vettraino M and Di Stefano G: Inhibition of lactate dehydrogenase activity as an approach to cancer therapy. Future Med Chem 6: 429-445, 2014.

21. Ždralević M, Brand A, Di Ianni L, Dettmer K, Reinders J, Singer K, Peter K, Schnell A, Bruss C, Decking SM, et al: Double genetic disruption of lactate dehydrogenases $\mathrm{A}$ and $\mathrm{B}$ is required to ablate the 'Warburg effect' restricting tumor growth to oxidative metabolism. J Biol Chem 293: 15947-15961, 2018.

22. Urbanska K and Orzechowski A: Unappreciated role of LDHA and LDHB to control apoptosis and autophagy in tumor cells. Int J Mol Sci 20: 2085, 2019.

23. Cui XG, Han ZT, He SH, Wu XD, Chen TR, Shao $\mathrm{CH}$, Chen DL, Su N, Chen YM, Wang T, et al: HIF $1 / 2 \alpha$ mediates hypoxia-induced LDHA expression in human pancreatic cancer cells. Oncotarget 8: 24840-24852, 2017.

24. Santama N, Ogg SC, Malekkou A, Zographos SE, Weis K and Lamond AI: Characterization of hCINAP, a novel coilin-interacting protein encoded by a transcript from the transcription factor TAFIID32 locus. J Biol Chem 280: 36429-36441, 2005.

25. Granneman S, Nandineni MR and Baserga SJ: The putative NTPase Fap7 mediates cytoplasmic 20S pre-rRNA processing through a direct interaction with Rps14. Mol Cell Biol 25: 10352-10364, 2005.

26. Zhang J, Bai D, Ma X, Guan J and Zheng X: hCINAP is a novel regulator of ribosomal protein-HDM2-p53 pathway by controlling NEDDylation of ribosomal protein S14. Oncogene 33: $246-254,2014$ 
27. Zhang J, Zhang F and Zheng X: Depletion of hCINAP by RNA interference causes defects in Cajal body formation, histone transcription, and cell viability. Cell Mol Life Sci 67: 1907-1918, 2010.

28. Xu R, Yu S, Zhu D, Huang X, Xu Y, Lao Y, Tian Y, Zhang J, Tang $\mathrm{Z}$, Zhang $\mathrm{Z}$, et al: hCINAP regulates the DNA-damage response and mediates the resistance of acute myelocytic leukemia cells to therapy. Nat Commun 10: 3812, 2019.

29. Ji Y, Yang C, Tang Z, Yang Y, Tian Y, Yao H, Zhu X, Zhang Z, Ji $J$ and Zheng $X$ : Adenylate kinase hCINAP determines self-renewal of colorectal cancer stem cells by facilitating LDHA phosphorylation. Nat Commun 8: 15308, 2017.

30. Iida A, Iwagawa T, Kuribayashi H, Satoh S, Mochizuki Y, Baba Y, Nakauchi H, Furukawa T, Koseki H, Murakami A and Watanabe S: Histone demethylase Jmjd3 is required for the development of subsets of retinal bipolar cells. Proc Natl Acad Sci USA 111: 3751-3756, 2014.

31. Livak KJ and Schmittgen TD: Analysis of relative gene expression data using real-time quantitative PCR and the 2(-Delta Delta C(T)) method. Methods 25: 402-408, 2001.

32. Brooks GA: Energy flux, lactate shuttling, mitochondrial dynamics, and hypoxia. Adv Exp Med Biol 903: 439-455, 2016.

33. Bonen A: The expression of lactate transporters (MCT1 and MCT4) in heart and muscle. Eur J Appl Physiol 86: 6-11, 2001.

34. Prech M, Marszalek A, Schröder J, Filas V, Lesiak M, Jemielity M, Araszkiewicz A and Grajek S: Apoptosis as a mechanism for the elimination of cardiomyocytes after acute myocardial infarction. Am J Cardiol 105: 1240-1245, 2010.

35. Bai D, Zhang J, Li T, Hang R, Liu Y, Tian Y, Huang D, Qu L, Cao X, Ji J and Zheng X: The ATPase hCINAP regulates $18 \mathrm{~S}$ rRNA processing and is essential for embryogenesis and tumour growth. Nat Commun 7: 12310, 2016.

36. Leong SP, Aktipis A and Maley C: Cancer initiation and progression within the cancer microenvironment. Clin Exp Metastasis 35: 361-367, 2018
37. Warburg O: On the origin of cancer cells. Science 123: 309-314, 1956.

38. Saddik M and Lopaschuk GD: Myocardial triglyceride turnover and contribution to energy substrate utilization in isolated working rat hearts. J Biol Chem 266: 8162-8170, 1991

39. Liochev SI: Reactive oxygen species and the free radical theory of aging. Free Radic Biol Med 60: 1-4, 2013.

40. Martinon F: Signaling by ROS drives inflammasome activation. Eur J Immunol 40: 616-619, 2010.

41. Hockenbery DM, Oltvai ZN, Yin XM, Milliman CL and Korsmeyer SJ: Bcl-2 functions in an antioxidant pathway to prevent apoptosis. Cell 75: 241-251, 1993.

42. Jeong D, Kim TS, Lee JW, Kim KT, Kim HJ, Kim IH and Kim IY: Blocking of acidosis-mediated apoptosis by a reduction of lactate dehydrogenase activity through antisense mRNA expression. Biochem Biophys Res Commun 289: 1141-1149, 2001.

43. Xu J, Xu X, Si L, Xue L, Zhang S, Qin J, Wu Y, Shao Y, Chen Y and Wang $X$ : Intracellular lactate signaling cascade in atrial remodeling of mitral valvular patients with atrial fibrillation. J Cardiothorac Surg 8: 34, 2013.

44. Gao C, Wang F, Wang Z, Zhang J and Yang X: Asiatic acid inhibits lactate-induced cardiomyocyte apoptosis through the regulation of the lactate signaling cascade. Int J Mol Med 38: 1823-1830, 2016

45. Qu L, Ji Y, Zhu X and Zheng X: hCINAP negatively regulates $\mathrm{NF}-\kappa \mathrm{B}$ signaling by recruiting the phosphatase PP1 to deactivate IKK complex. J Mol Cell Biol 7: 529-542, 2015.

46. Yan Y, Yuan X, Xue C and He Y: Human coilin interacting nuclear ATPase protein in cancer: Uncovering new insights into pathogenesis and therapy. Am J Transl Res 12: 4051-4058, 2020.

This work is licensed under a Creative Commons Attribution-NonCommercial-NoDerivatives 4.0 International (CC BY-NC-ND 4.0) License. 